\title{
Computational Modeling of the Virucidal Inhibition Mechanism for Broad- Spectrum Antiviral Nanoparticles and HPV16 Capsid Segments
}

\author{
Parth Chaturvedi, Payam Kelich, Tara A. Nitka, Lela Vuković ${ }^{\star}$ \\ Department of Chemistry and Biochemistry, University of Texas at EI Paso, EI Paso, TX 79968, USA
}

"Email: Lvvkovic@utep.edu

\section{Electronic Supporting Information (ESI)}


Table S1. Sizes and numbers of atoms in the simulated systems.

\begin{tabular}{|ccc|}
\hline control systems (no NP) & unit cell size (relaxed) & number of atoms \\
\hline 2 pentamers & $244 \times 244 \times 142 \AA$ & 852,741 \\
3 pentamers & $267 \times 268 \times 156 \AA$ & $1,136,444$ \\
4 pentamers & $367 \times 307 \times 162 \AA$ & $1,851,200$ \\
& & \\
\hline systems with NP & unit cell size (relaxed) & number of atoms \\
\hline 2 pentamers & $239 \times 244 \times 163 \AA$ & 960,769 \\
3 pentamers & $294 \times 287 \times 163 \AA$ & $1,387,902$ \\
4 pentamers & $357 \times 297 \times 162 \AA$ & $1,742,124$ \\
\hline
\end{tabular}

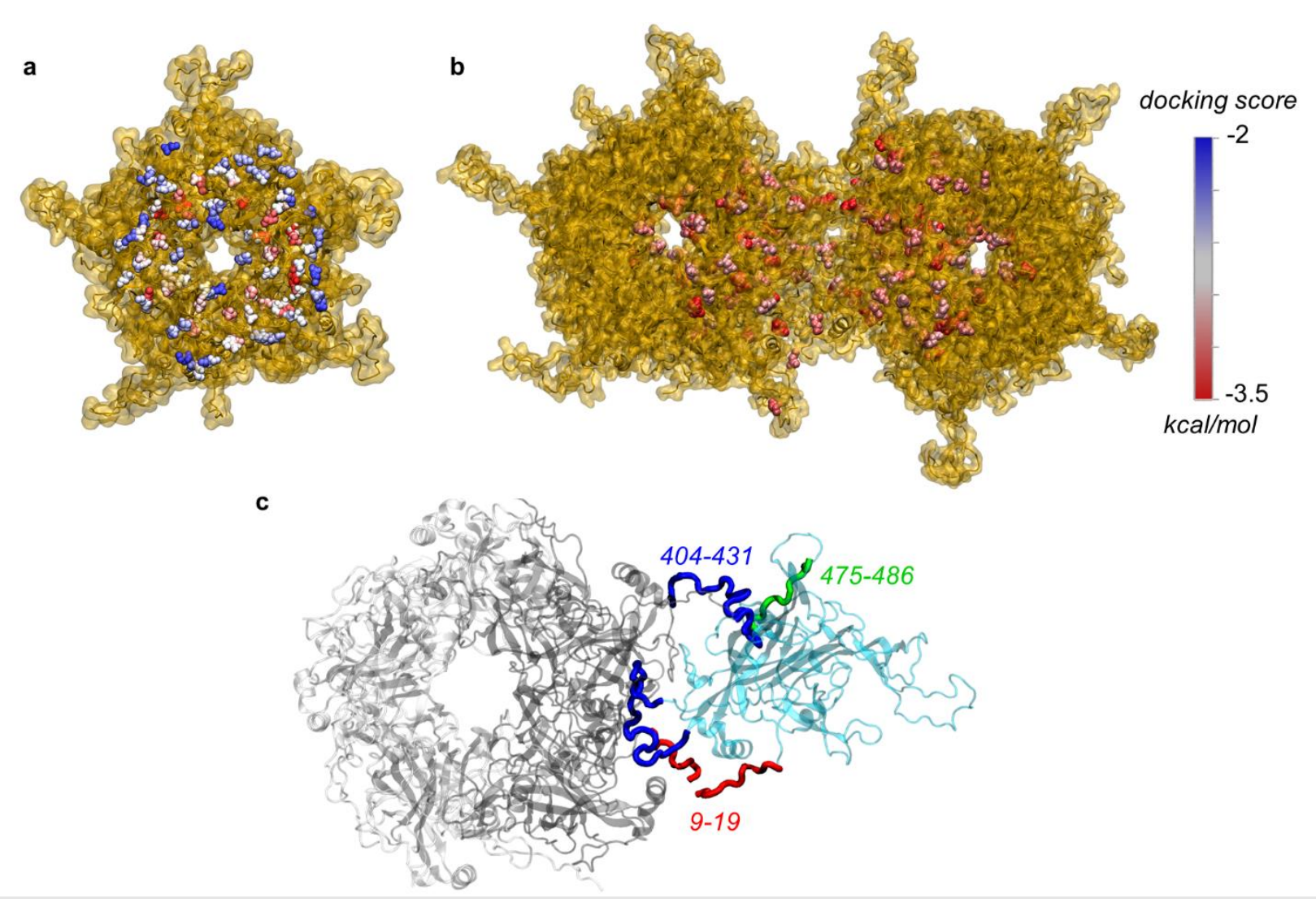

Figure S1. The results of the docking calculations and the structural details at pentamerpentamer interfaces. a) Structures of ligands $\left(\mathrm{CH}_{3} \mathrm{CH}_{2} \mathrm{SO}_{3}{ }^{-}\right)$docked on the surface of a single HPV L1 protein, colored according to the docking score. b) Structures of ligands docked on the surface of a pair of L1 proteins, colored according to the docking score. In this panel, only the ligands with the lowest docking scores are shown. c) Pentamer-pentamer interfaces reported in the pdbID: 3J6R ${ }^{1}$ structure. Structural models of our systems were based on L1 protein crystal structures (PDB: 5W1O) fitted into capsid segment structures, based on pdbID: 3J6R. Structures of the residues missing in the $5 \mathrm{~W} 10$ structure, namely residues 9-19, 404-431, and 475-486, highlighted above, were reconstructed based on their structures reported in pdbID: $3 J 6 R$. 
a
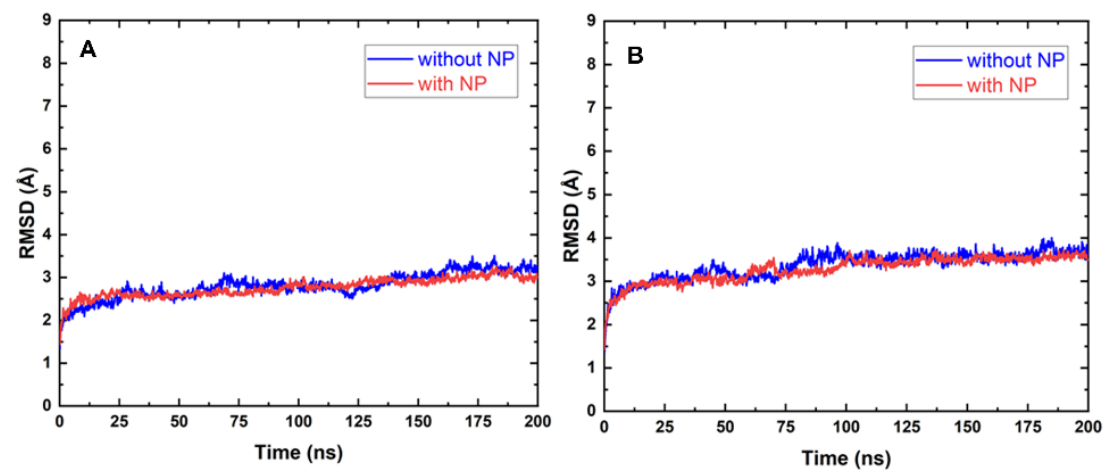

b
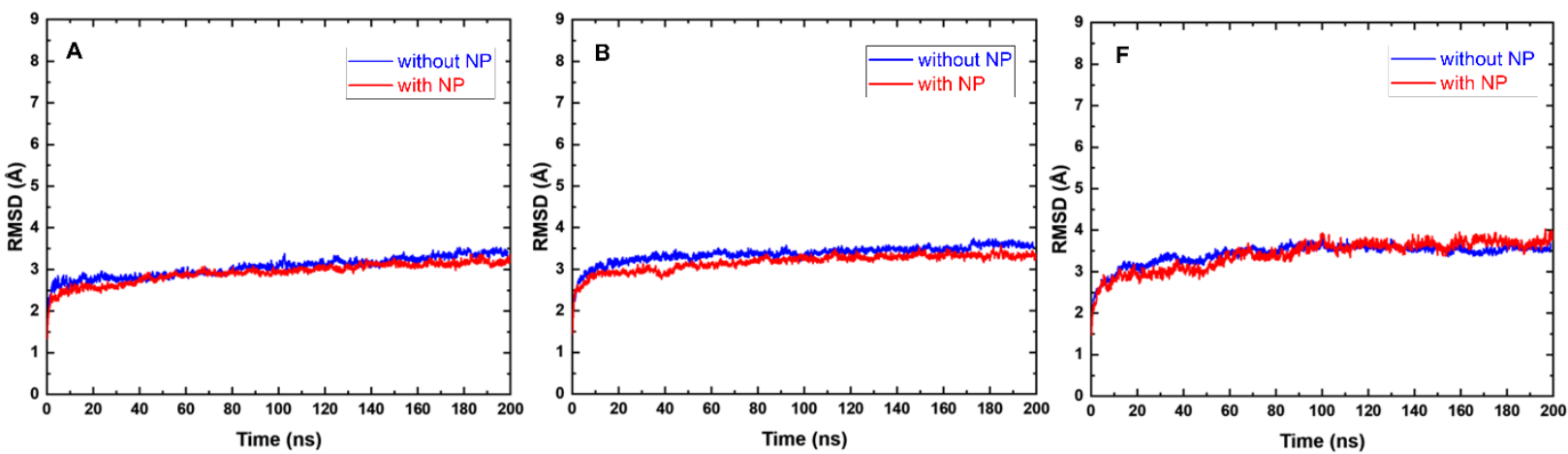

Figure S2. RMSDs of L1 pentamers (parts with defined secondary structures, consisting of $\alpha$ helices and $\beta$-sheets) observed during MD simulations for: a) two-pentamer systems, and $b$ ) three-pentamer systems. 

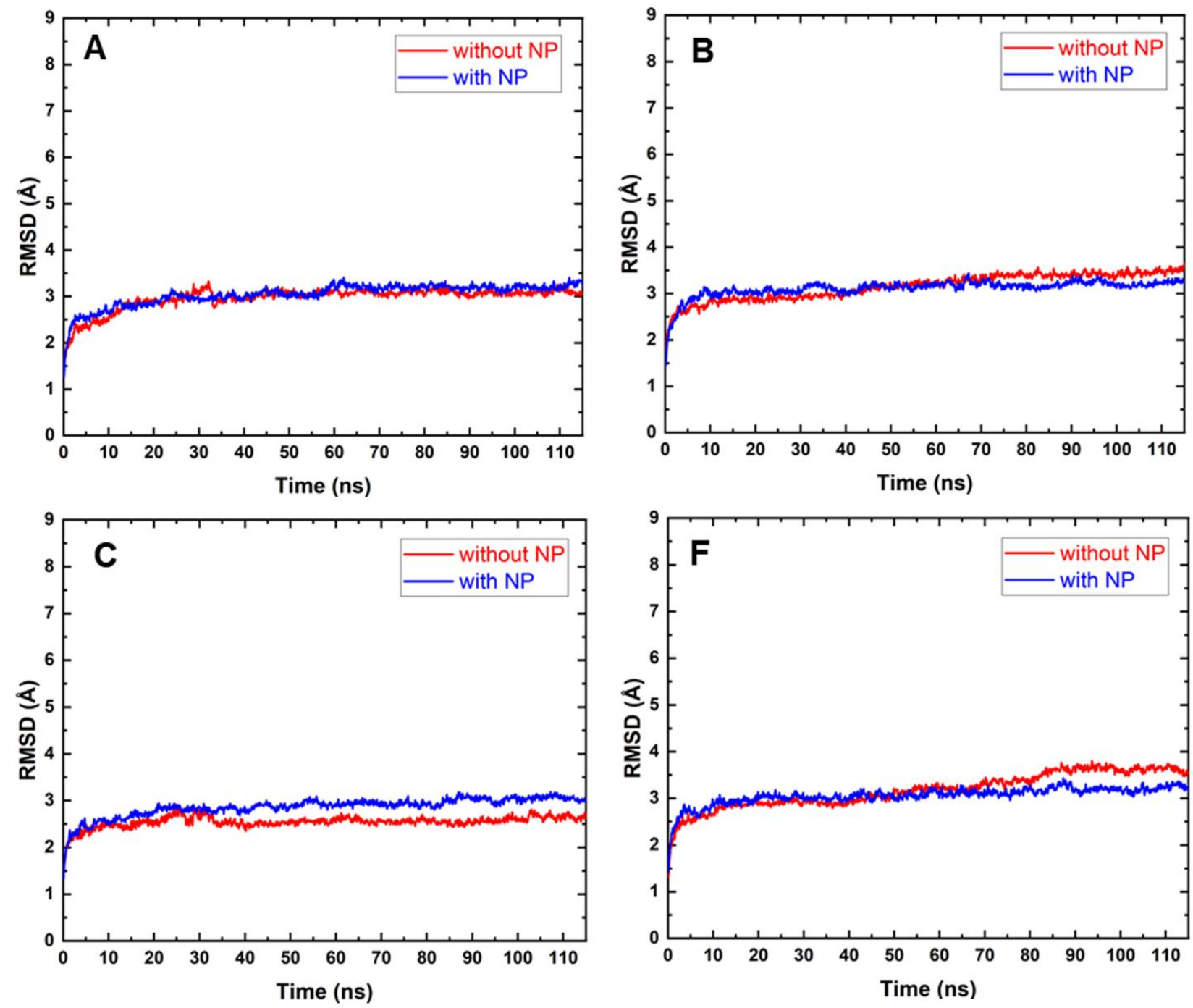

Figure S3. RMSDs of L1 pentamers (parts with defined secondary structures, consisting of $\alpha$ helices and $\beta$-sheets) observed during MD simulations for four-pentamer systems. 

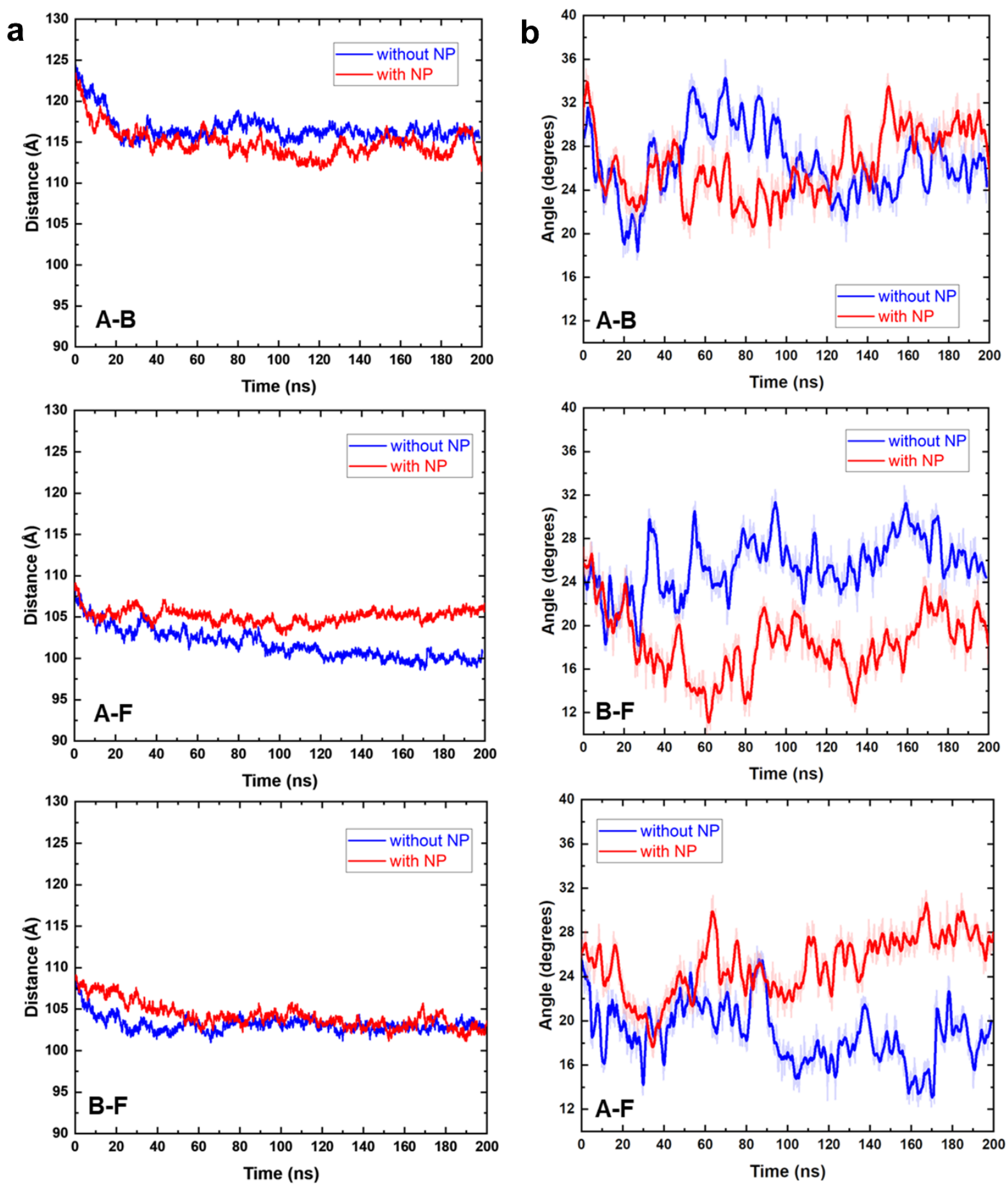

Figure S4. a) Distances between centers of mass (COMs) of all two L1 pentamer pairs in threepentamer systems observed during the first 200 ns of MD simulations. b) Angles between all two L1 pentamer pairs in three-pentamer systems observed during the first 200 ns of MD simulations. 

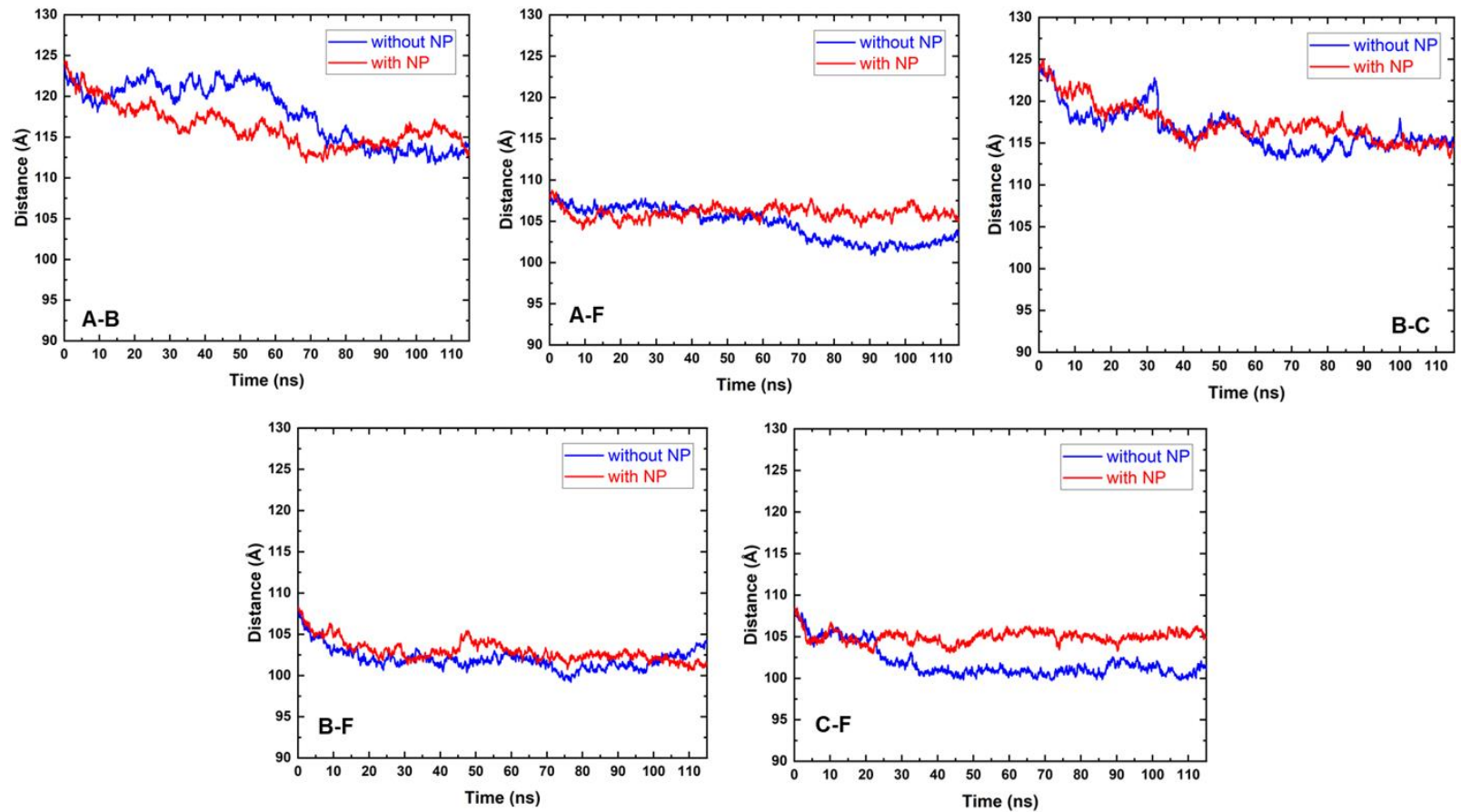

Figure S5. Distances between COMs of all two L1 pentamer pairs in four-pentamer systems observed during MD simulations.
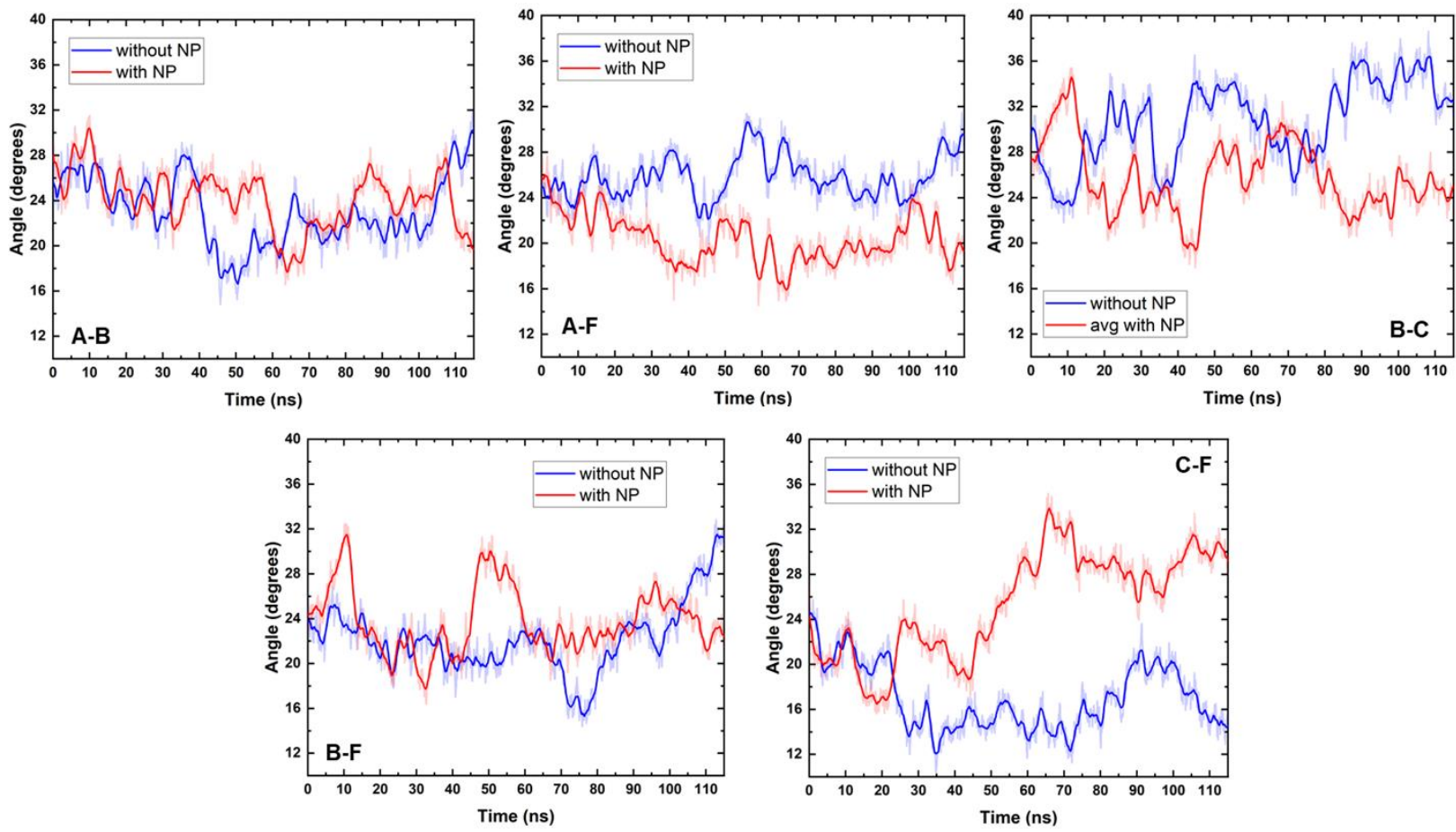

Figure S6. Angles between all two L1 pentamer pairs in four-pentamer systems observed during MD simulations. 

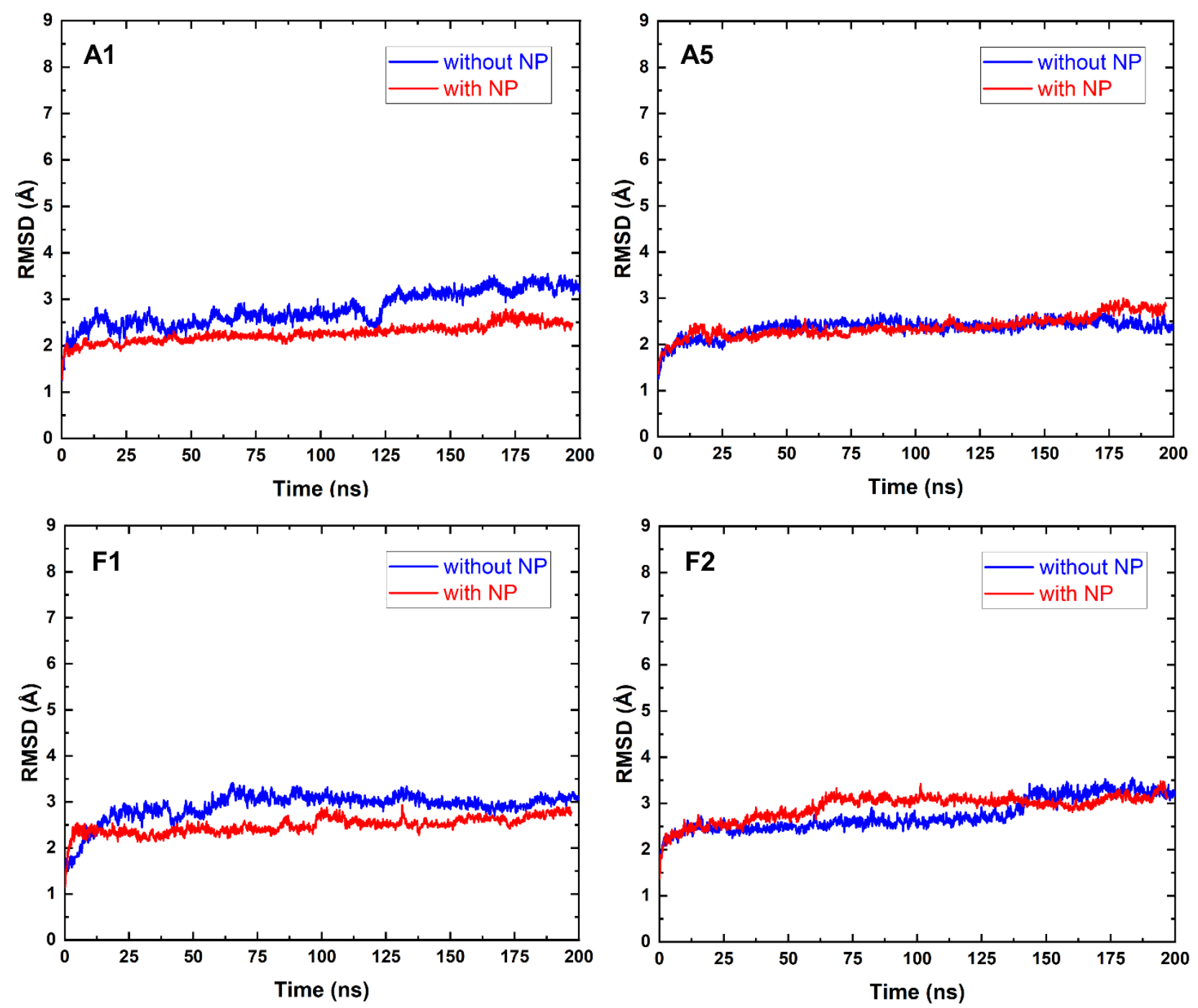

Figure S7. RMSDs of four NP-binding chains (their parts with defined secondary structures, consisting of $\alpha$-helices and $\beta$-sheets) within two-pentamer systems observed during MD simulations. 

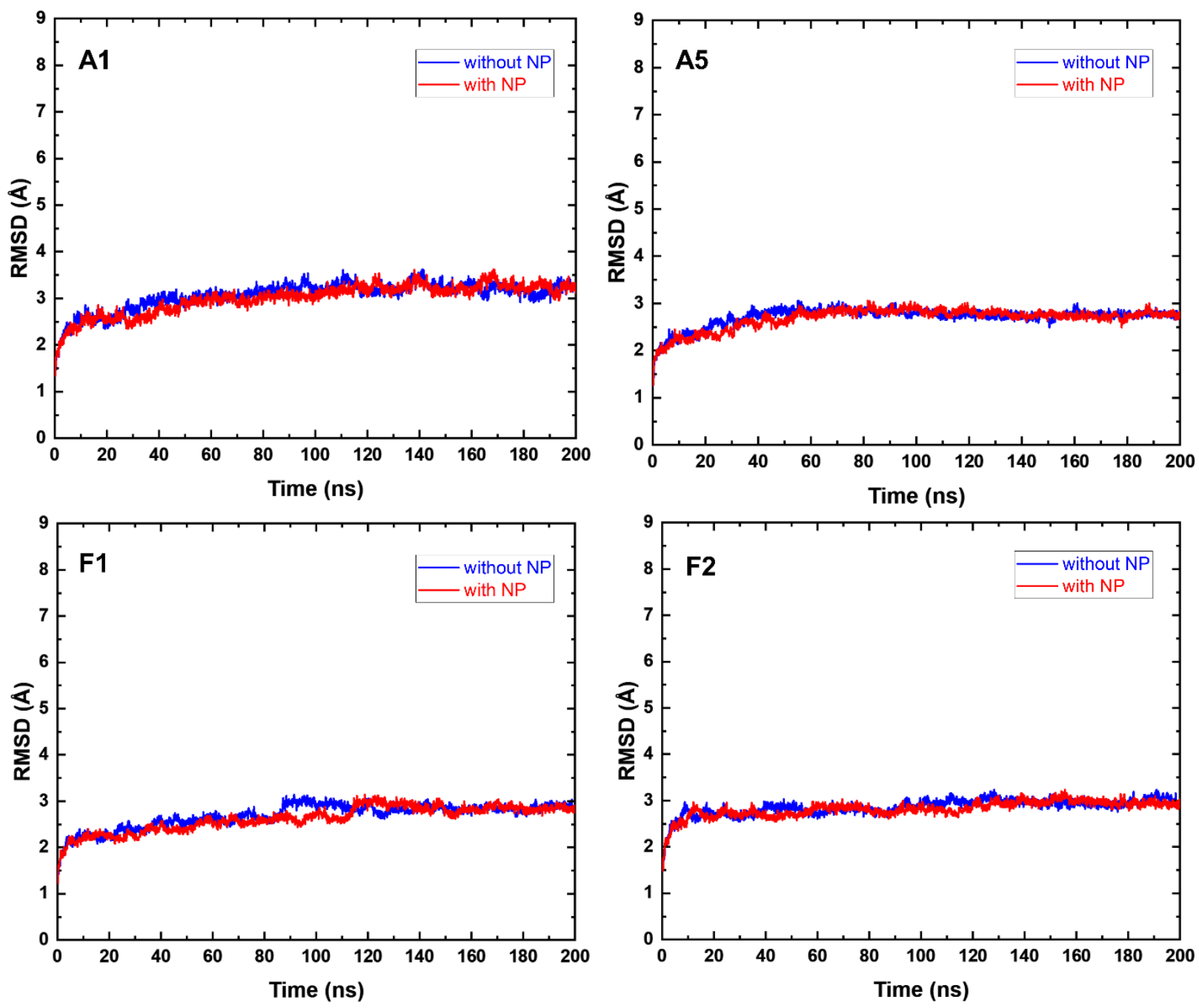

Figure S8. RMSDs of four NP-binding chains (their parts with defined secondary structures, consisting of $\alpha$-helices and $\beta$-sheets) within three-pentamer systems observed during MD simulations. 

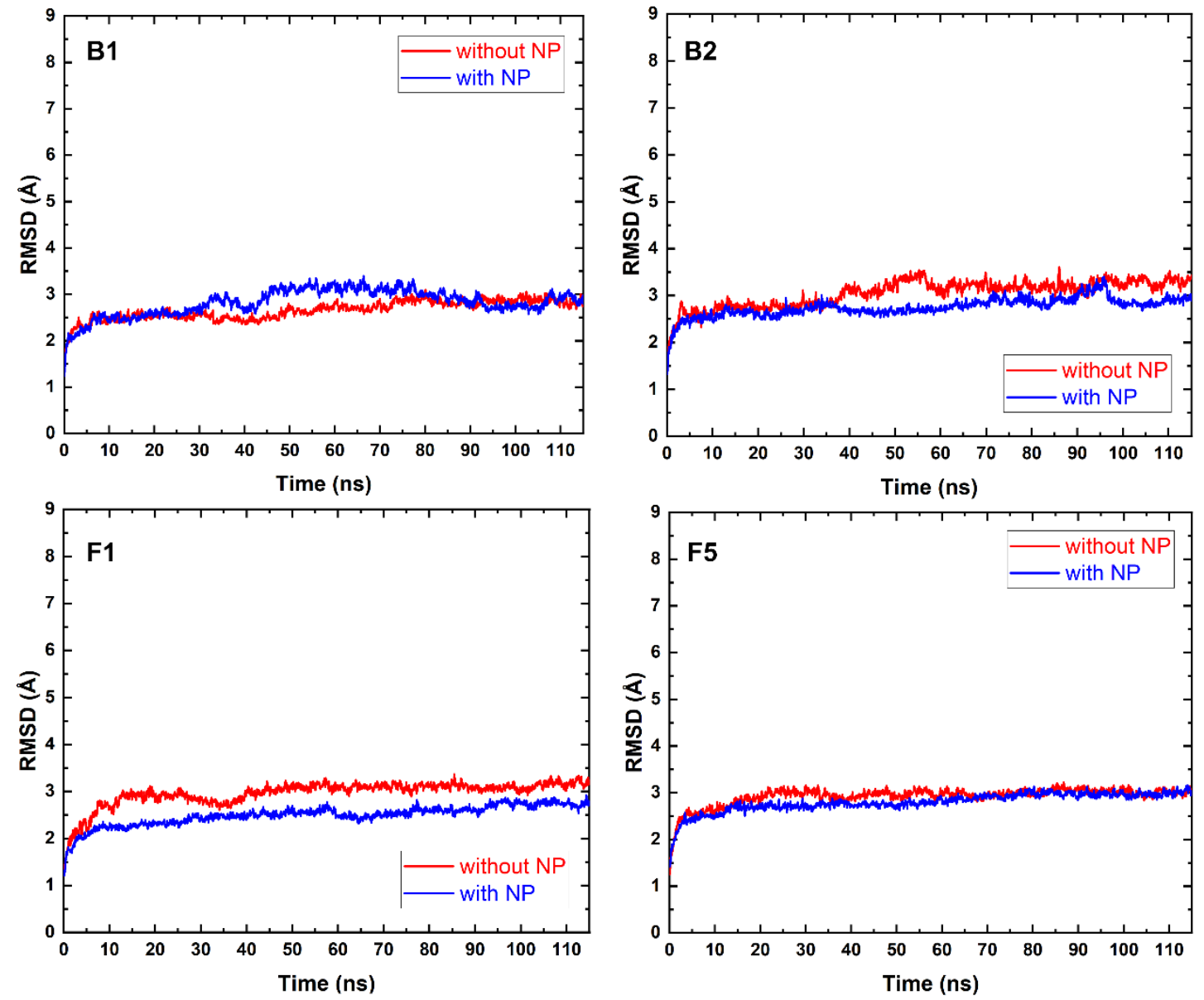

Figure S9. RMSDs of four NP-binding chains (their parts with defined secondary structures, consisting of $\alpha$-helices and $\beta$-sheets) within four-pentamer systems observed during MD simulations. 
a

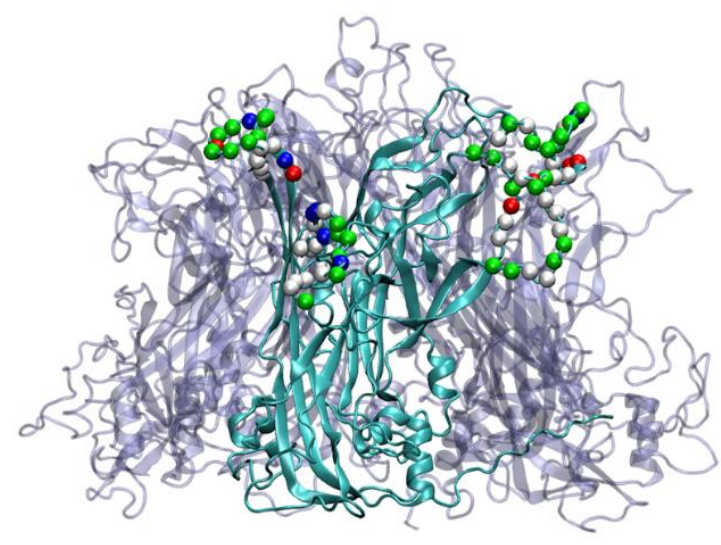

C

Residue index

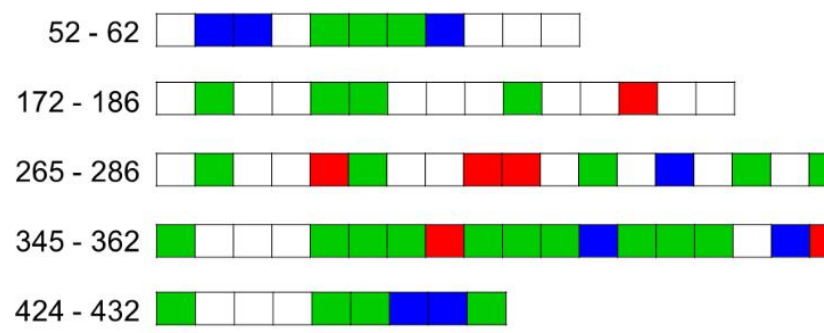

b

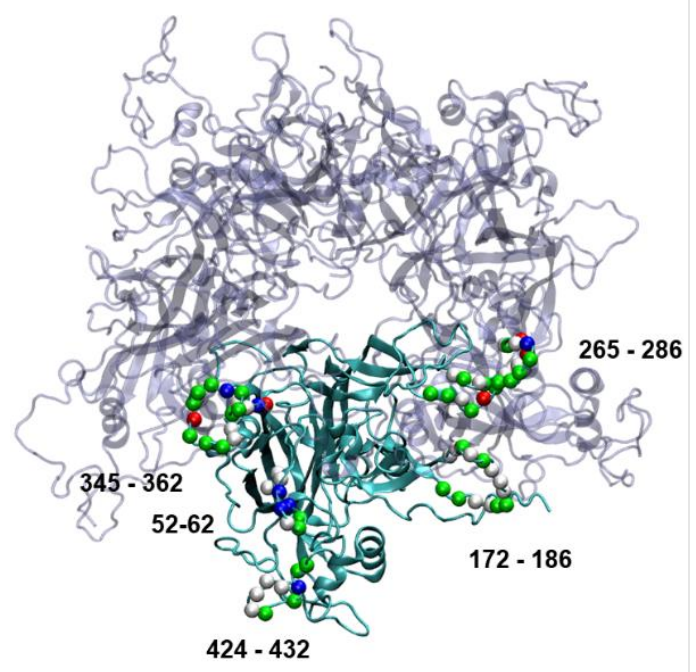

Figure S10. a) Side view and b) top view of two typical chains (cyan) of the pentamer with residues interacting with NP, shown as spheres that are colored according to their residue types. c) Residues interacting with the NP shown according to their respective types. 
a
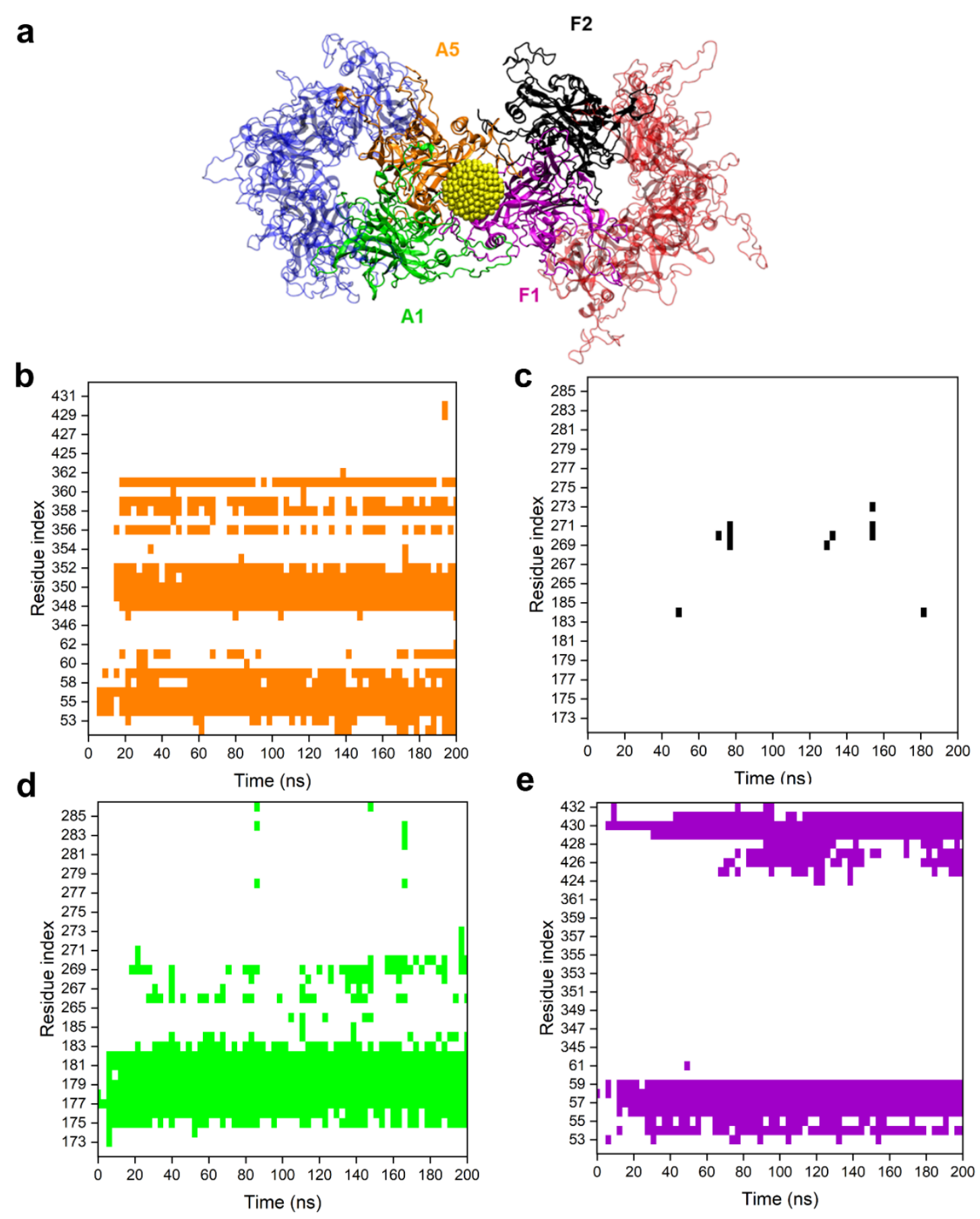

Figure S11. Interactions of MUS:OT NP at the interface of two L1 pentamers in the threepentamer system. a) A snapshot of the NP interacting at the interface of two pentamers, shown in faded blue and red. The NP interacts with two distinct chains of both $A$ and $F$ pentamers, labeled as A1 (green), A5 (orange), F1 (purple) and F2 (black). The NP gold core is shown in yellow, and MUS and OT ligands are not shown for clarity. b-e) Contact maps of residues of the four chains of three-pentamer systems found to interact with NP over the course of the simulation. Colors in the contact maps match the colors of the four chains labeled within pentamers in panel a (A5 - orange, F2 - black, A1 - green, and F1 - purple). White regions in contact maps denote no contact. 
a

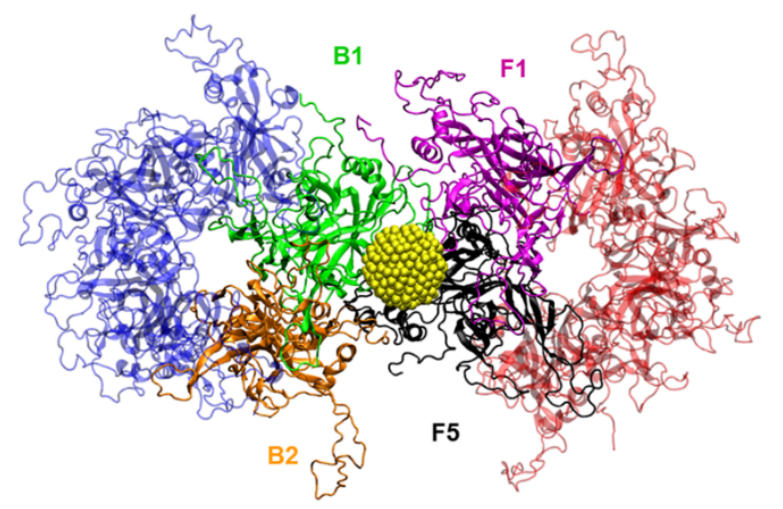

b

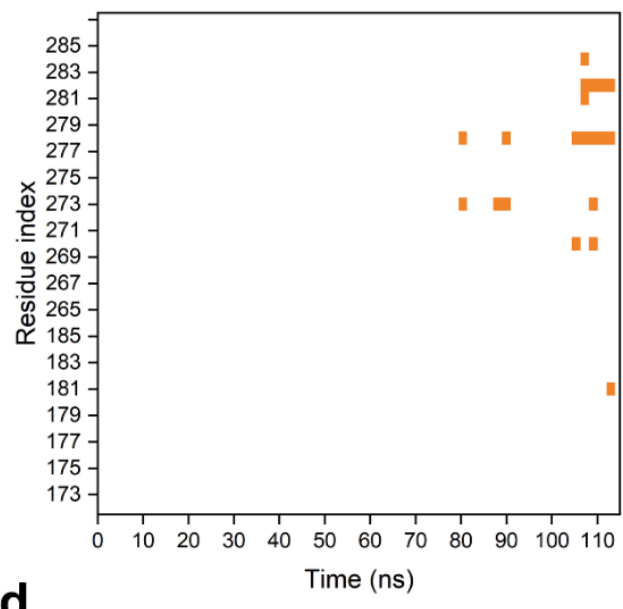

C

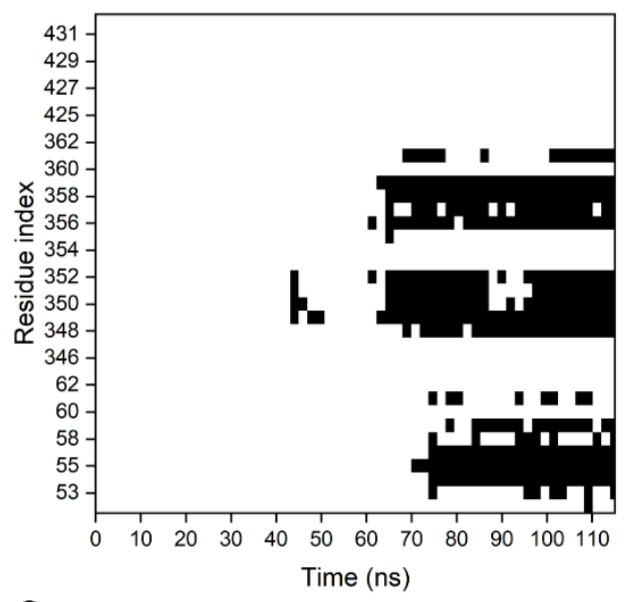

e
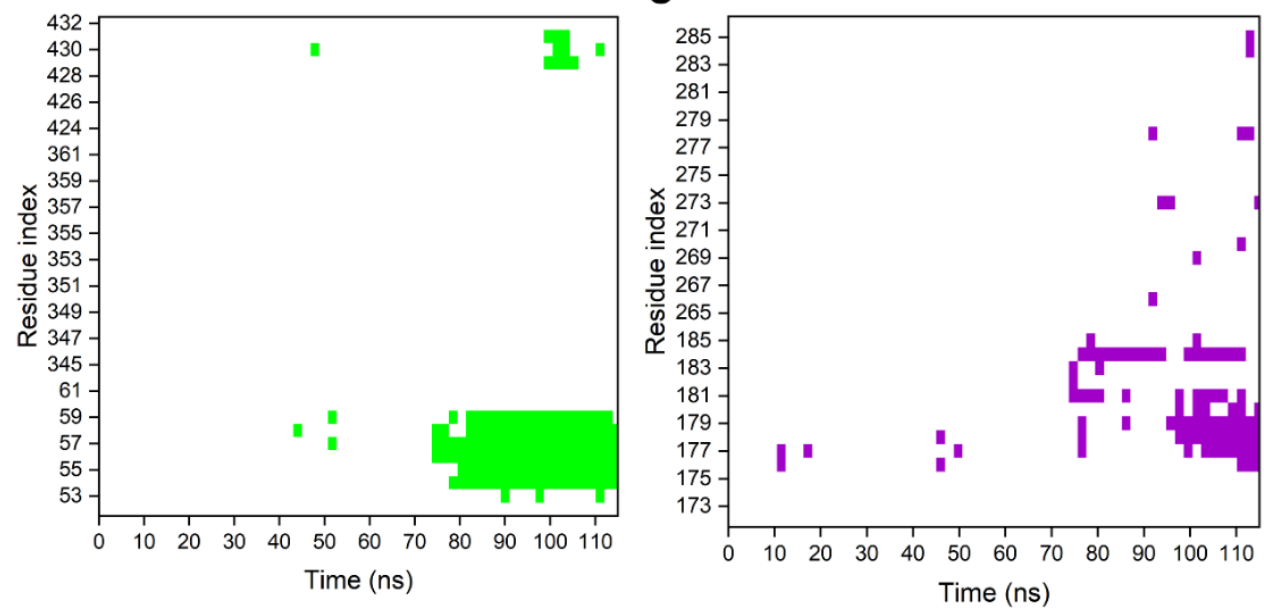

Figure S12. Interactions of MUS:OT NP at the interface of two L1 pentamers in the fourpentamer system. a) A snapshot of the NP interacting at the interface of two pentamers, shown in faded blue and red. The NP interacts with two distinct chains of both $B$ and $F$ pentamers, labeled as B1 (green), B2 (orange), F1 (purple) and F5 (black). The NP gold core is shown in yellow, and MUS and OT ligands are not shown for clarity. b-e) Contact maps of residues of the four chains of four-pentamer systems found to interact with NP over the course of the simulation. Colors in the contact maps match the colors of the four chains labeled within pentamers in panel a. White regions in contact maps denote no contact. 
a
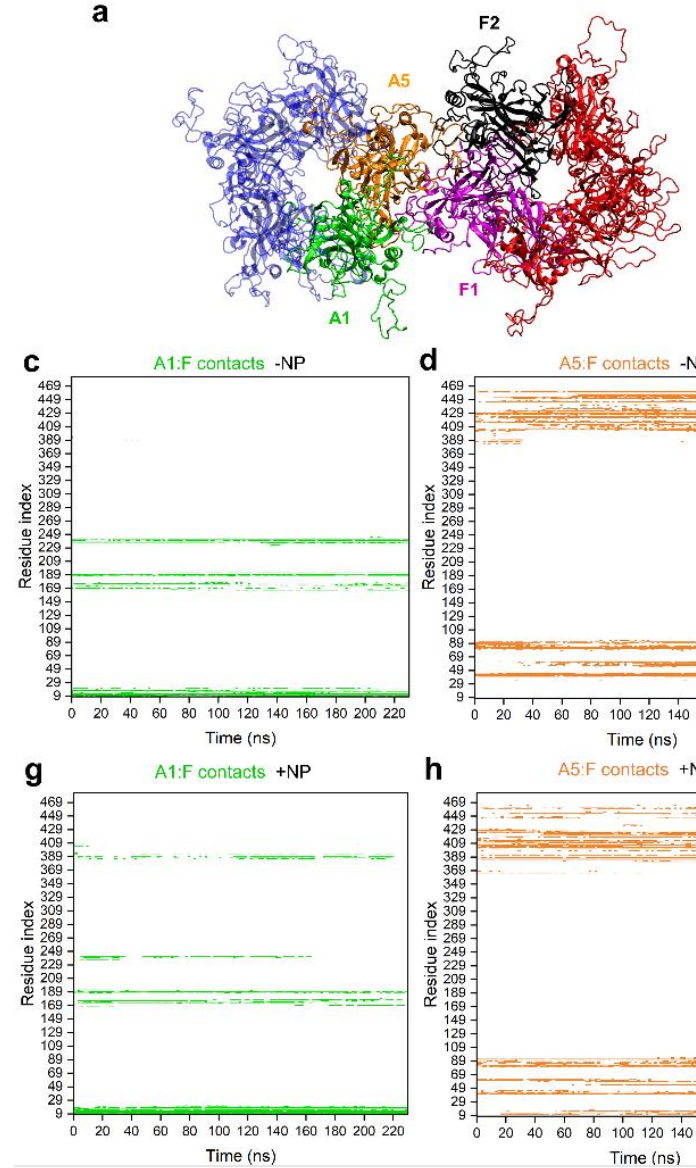

d

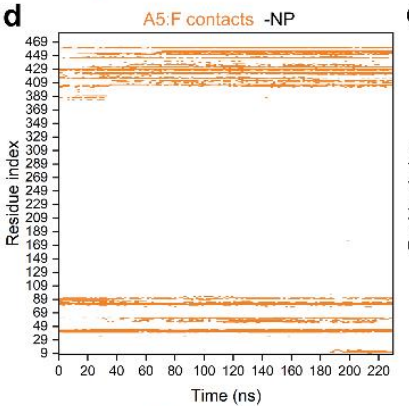

h

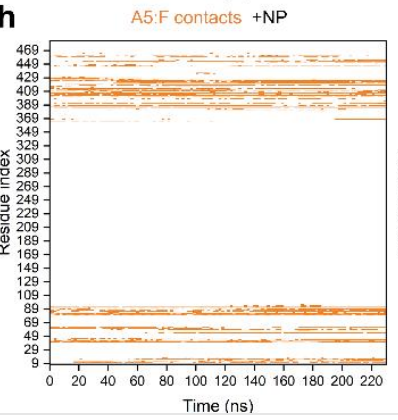

b

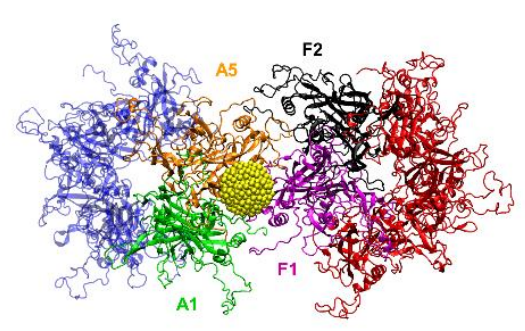

e
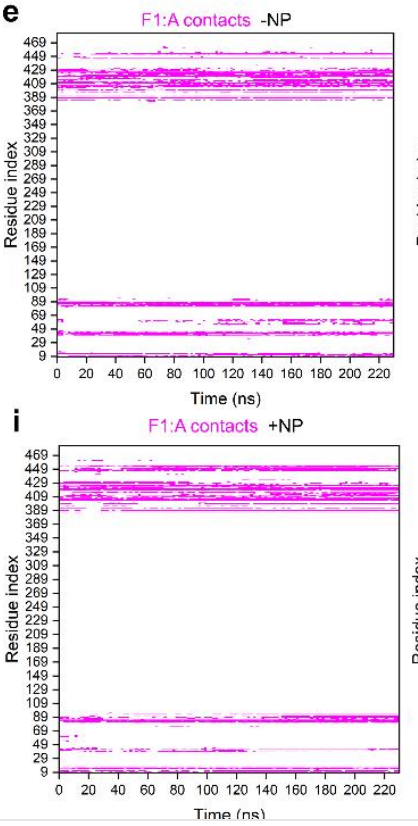

f
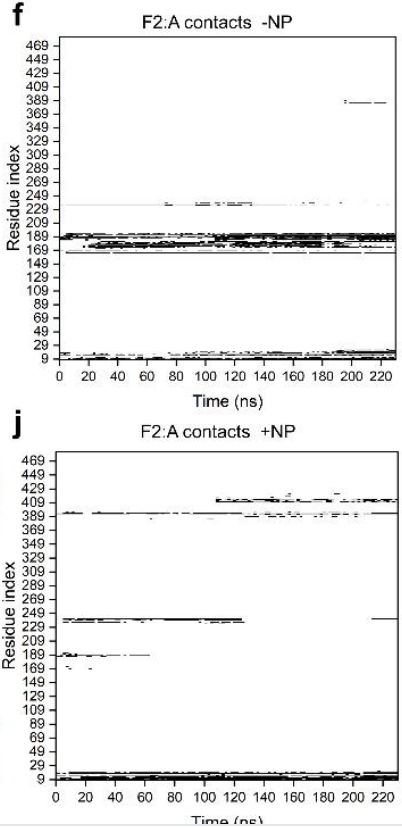

Figure S13. a) A snapshot of the two interacting pentamers in three-pentamer system. b) A snapshot of the NP interacting at the interface of the same two pentamers within the threepentamer system. Chains of neighboring pentamers interacting with each other are labeled in distinct colors. The NP gold core is shown in yellow, and MUS and OT ligands are not shown for clarity. c-f) ) Contact maps of A1 and A5 chain residues in contact with F pentamer and $F 1$ and $\mathrm{F} 2$ chain residues in contact with A pentamer within three-pentamer systems in the absence of MUS:OT NP over the course of the simulation. g-j) Contact maps of A1 and A5 chain residues in contact with $\mathrm{F}$ pentamer and $\mathrm{F} 1$ and $\mathrm{F} 2$ chain residues in contact with A pentamer within fourpentamer systems in the presence of MUS:OT NP over the course of the simulation. 

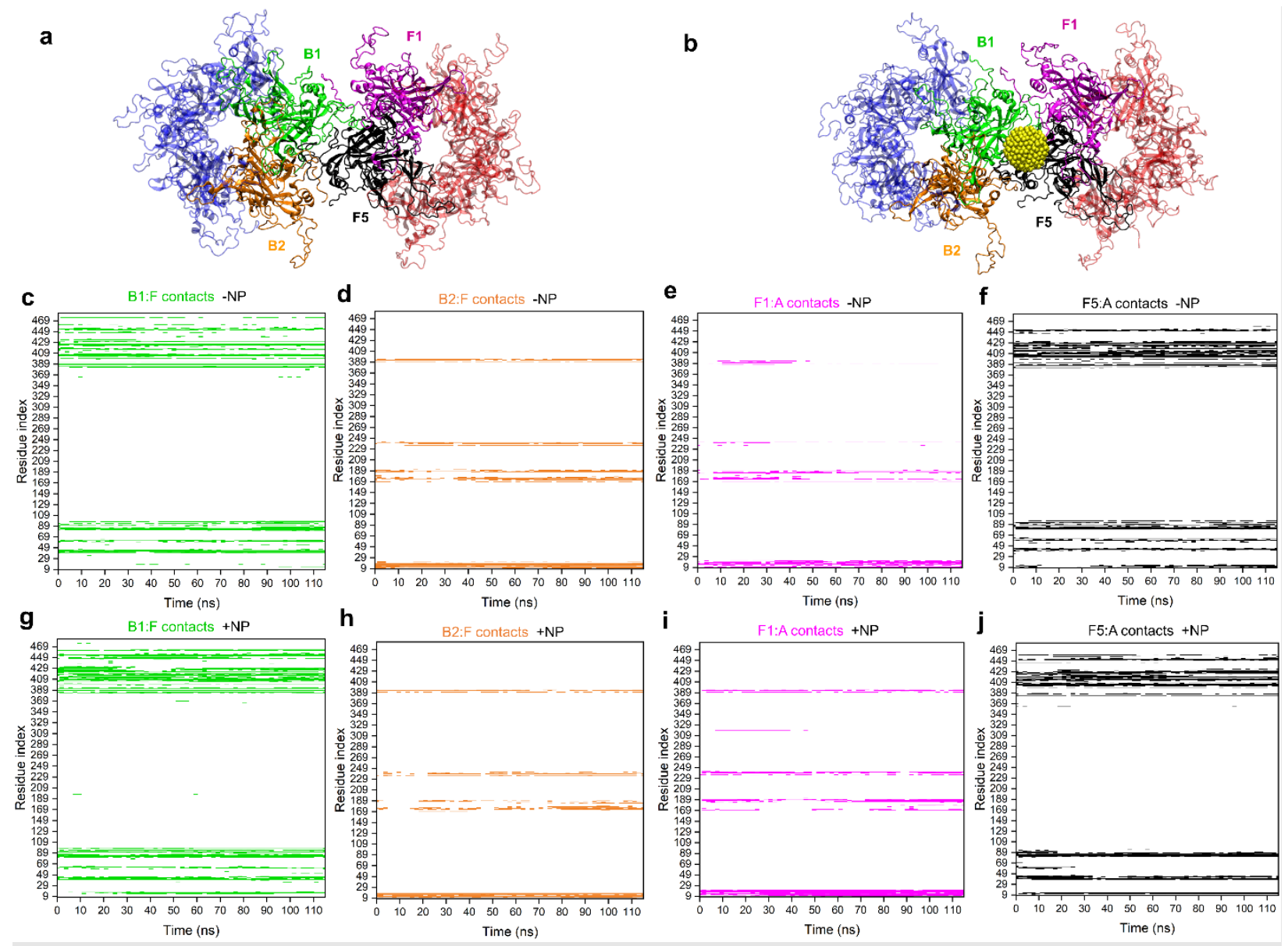

Figure S14. a) A snapshot of two interacting pentamers in the four-pentamer system. b) A snapshot of the NP interacting at the interface of the same two pentamers within the fourpentamer system. Chains of neighboring pentamers interacting with each other are labeled in distinct colors. The NP gold core is shown in yellow, and MUS and OT ligands are not shown for clarity. C-f) ) Contact maps of B1 and B2 chain residues in contact with F pentamer and $\mathrm{F} 1$ and F5 chain residues in contact with A pentamer within four-pentamer systems in the absence of MUS:OT NP over the course of the simulation. g-j) Contact maps of B1 and B2 chain residues in contact with $\mathrm{F}$ pentamer and $\mathrm{F} 1$ and $\mathrm{F} 5$ chain residues in contact with $\mathrm{B}$ pentamer within fourpentamer systems in the presence of MUS:OT NP over the course of the simulation. 

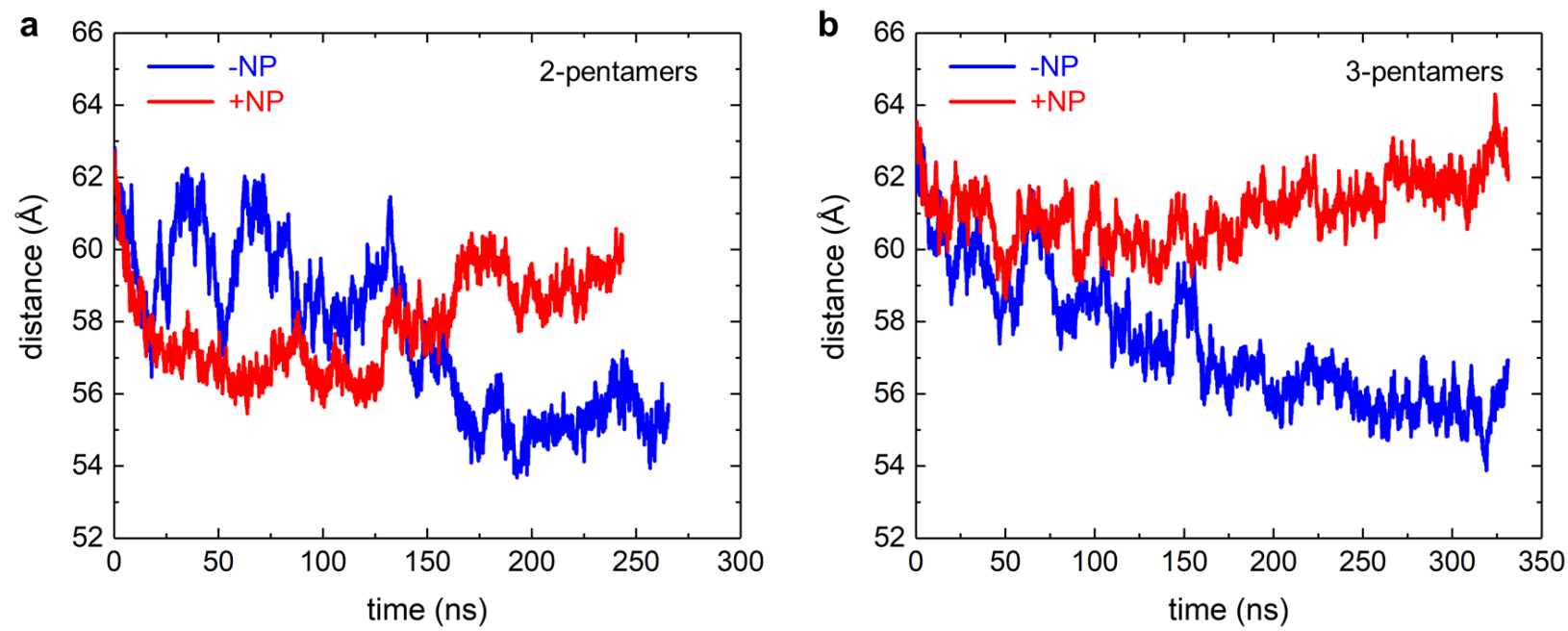

Figure S15. Distances between chains of $A$ and $F$ pentamers that are in direct contact in HPV capsid segments alone and when interacting with MUS:OT NP. a) Distance between COM of $A 1, A 5$ chains from the COM of $F 1, F 2$ chains of $A$ and $F$ pentamers alone and when interacting with NP for two-pentamer segment systems. b) Distance between A1, A5 chains from F1, F2 chains of $A$ and $F$ pentamers alone and when interacting with NP for three-pentamer segment systems.

Table S2. Distances and angles in two- and three-pentamer systems with and without NP, averaged over the last 100 ns of trajectories.

\begin{tabular}{|ccc|}
\hline system & distance $(\mathbf{\AA})$ & angle $\left(^{\circ}\right)$ \\
\hline 2-pentamer & 99.61 & 22.08 \\
2-pentamer + NP & 104.06 & 27.09 \\
3-pentamer & 100.11 & 17.84 \\
3-pentamer + NP & 105.78 & 27.41 \\
\hline
\end{tabular}

\section{References}

1. Cardone, G., Moyer, A. L., Cheng, N., Thompson, C. D., Dvoretzky, I., Lowy, D. R., Schiller, J. T., Steven, A. C., Buck, C. B., Trus, B. L. \& Imperiale, M. J., "Maturation of the Human Papillomavirus 16 Capsid," MBio 5, e01104-14 (2014). DOI:

10.1128/mBio.01104-14 